\title{
A nonlinear model dynamics for closed-system, constrained, maximal-entropy-generation relaxation by energy redistribution
}

\author{
Gian Paolo Beretta \\ Università di Brescia, via Branze 38, 25123 Brescia, Italy \\ beretta@unibs.it \\ (October 13, 2018)
}

We discuss a nonlinear model for the relaxation by energy redistribution within an isolated, closed system composed of non-interacting identical particles with energy levels $e_{i}$ with $i=1,2, \ldots, N$. The time-dependent occupation probabilities $p_{i}(t)$ are assumed to obey the nonlinear rate equations $\tau d p_{i} / d t=-p_{i} \ln p_{i}-\alpha(t) p_{i}-\beta(t) e_{i} p_{i}$ where $\alpha(t)$ and $\beta(t)$ are functionals of the $p_{i}(t)$ 's that maintain invariant the mean energy $E=\sum_{i=1}^{N} e_{i} p_{i}(t)$ and the normalization condition $1=\sum_{i=1}^{N} p_{i}(t)$. The entropy $S(t)=-k_{\mathrm{B}} \sum_{i=1}^{N} p_{i}(t) \ln p_{i}(t)$ is a non-decreasing function of time until the initially nonzero occupation probabilities reach a Boltzmann-like canonical distribution over the occupied energy eigenstates. Initially zero occupation probabilities, instead, remain zero at all times. The solutions $p_{i}(t)$ of the rate equations are unique and welldefined for arbitrary initial conditions $p_{i}(0)$ and for all times. Existence and uniqueness both forward and backward in time allows the reconstruction of the ancestral or primordial lowest entropy state. By casting the rate equations not in terms of the $p_{i}$ 's but of their positive square roots $\sqrt{p_{i}}$, they unfold from the assumption that time evolution is at all times along the local direction of steepest entropy ascent or, equivalently, of maximal entropy generation. These rate equations have the same mathematical structure and basic features of the nonlinear dynamical equation proposed in a series of papers ended with G. P. Beretta, Found. Phys. 17, 365 (1987) and recently rediscovered in S. Gheorghiu-Svirschevski, Phys. Rev. A 63, 022105 and 054102 (2001). Numerical results illustrate the features of the dynamics and the differences with the rate equations recently considered for the same problem in $\mathrm{M}$. Lemanska and Z. Jaeger, Physica D 170, 72 (2002). We also interpret the functionals $k_{\mathrm{B}} \alpha(t)$ and $k_{\mathrm{B}} \beta(t)$ as nonequilibrium generalizations of the thermodynamic-equilibrium Massieu characteristic function and inverse temperature, respectively.

\section{INTRODUCTION}

Much work has appeared in recent years on the study of entropy-generating irreversible nonequilibrium dynamics. Limited discussions of previous work is found in [1-3] and references therein, but no thorough critical review of the subject is available, although it would be very helpful to provide proper acknowledgement of pioneering work, avoid 'rediscoveries' such as in [4] and outline the different frameworks, motivations, approaches and controversial aspects. To be sure, recent discussions $[2,4-6]$ on possible fundamental tests of standard unitary quantum mechanics, related to the existence of 'spontaneous decoherence' at the microscopic level, and on understanding and predicting decoherence in important future applications [7] involving nanometric devices, fast switching times, clock synchronization, superdense coding, quantum computation, teleportation, quantum cryptography, etc. show that the subject of irreversible nonequilibrium dynamics is by no means settled.

It is not the purpose of this paper to attempt such a difficult review, nor to address the related fundamental issues lurking beneath interpretation (see, e.g., [8-10]). Rather we wish to address the model problem recently outlined in [1].

This model may prove useful to complement various historical and contemporary efforts to extend linear Markovian theories of dissipative phenomena and relaxation based on master equations, Lindblad and Langevin equations, to the nonlinear and far nonequilibrium domain. For example, spectroscopic studies of the effects of vibrational relaxation on line shapes of twolevel electronic transitions cannot be regularized under the Markovian approximations so that various nonlinear approaches are being developed and tested [11] in some cases at the expense of giving up preservation of (complete) positivity [12] or hermiticity [13] of the (reduced) density operator.

Again, it is not our purpose here to review the literature of these specific potential applications of our model dynamics, nor to apply it explicitly to particular examples. Rather we wish to focus on illustrating its general features (including preservation of positivity and hermiticity at all times, even backwards) that make it a good candidate (that is, compatible with all reasonable requirements imposed by thermodynamic principles [14]) of extensions of the traditional linear master equations for open system dynamics, capable to include the description of nonlinear spontaneous relaxation within the system (even if isolated) by energy redistribution between the occupied levels.

We consider an isolated, closed system composed of non-interacting identical particles with single-particle energy levels $e_{i}$ with $i=1,2, \ldots, N$ where $N$ is assumed finite for simplicity and the $e_{i}$ 's are repeated in case of degeneracy. We restrict our attention on the class of dilute-Boltzmann-gas states in which the particles are independently distributed among the $N$ (possibly degenerate) one-particle energy eigenstates. In density oper- 
ator language, this is tantamount to restricting the attention on the subset of one-particle density operators that are diagonal in the representation which diagonalizes the one-particle Hamiltonian operator. We denote by $p_{i}$ the occupation probability of the $i$-th eigenstate, so that the per-particle mean energy, normalization and entropy functionals are given by the relations

$$
\begin{aligned}
& E(\mathbf{p})=\sum_{i=1}^{N} e_{i} p_{i}, \quad U(\mathbf{p})=\sum_{i=1}^{N} p_{i}, \\
& S(\mathbf{p})=-k_{\mathrm{B}} \sum_{i=1}^{N} p_{i} \ln p_{i},
\end{aligned}
$$

where $\mathbf{p}$ denotes the vector of $p_{i}$ 's, the Boltzmann constant $k_{\mathrm{B}}$ may be used to nondimensionalize $S$ (or we may assume for simplicity $k_{\mathrm{B}}=1$ unit of entropy), and of course $U(\mathbf{p})=1$ for any normalized distribution $\mathbf{p}$.

As is well known, for a given value of $E$, the thermodynamic-equilibrium canonical distribution

$$
p_{j}^{\mathrm{se}}(E)=\frac{\exp \left(-\beta^{\mathrm{se}}(E) e_{j}\right)}{\sum_{i=1}^{N} \exp \left(-\beta^{\mathrm{se}}(E) e_{i}\right)}
$$

has inverse temperature $\beta^{\mathrm{se}}(E)=1 / k_{\mathrm{B}} T(E)$ and maximal entropy $S^{\mathrm{se}}(E)=-k_{\mathrm{B}} \sum_{i=1}^{N} p_{i}^{\mathrm{se}}(E) \ln p_{i}^{\mathrm{se}}(E)$.

We are interested in studying the dynamics of a nonequilibrium distribution obtained, for example, by exciting some energy eigenstates. As suggested in [1], a way to alter the distribution is to repopulate (e.g., by selective laser heating) or depopulate (in principle, by selective cooling or resonance fluorescence) a subset of eigenstates. This is described by multiplying each $p_{j}$ by a perturbation factor $f_{j} \geq 0$ (with $j=1,2, \ldots, N$ ) (repopulation $f_{j}>1$, depopulation $f_{j}<1$ ) and then renormalizing, to yield the perturbed nonequilibrium distribution

$$
\widetilde{p}_{j}=\frac{f_{j} p_{j}^{\mathrm{se}}(E)}{\sum_{i=1}^{N} f_{i} p_{i}^{\mathrm{se}}(E)} .
$$

Of course, in general the perturbed distribution has a different mean energy $\widetilde{E}=\sum_{i=1}^{N} e_{i} \widetilde{p}_{i}$ and different entropy $\widetilde{S}=-k_{\mathrm{B}} \sum_{i=1}^{N} \widetilde{p}_{i} \ln \widetilde{p}_{i}$. However, a proper choice of the perturbation factors $f_{i}$ may maintain $\widetilde{E}=E$, in which case $\widetilde{S}<S^{\text {se }}(E)$ (see Section VII).

To describe the relaxation towards the new target canonical equilibrium distribution $\mathbf{p}^{\mathrm{se}}(\widetilde{E})$, the dynamical equation proposed in [1] is, for $j=1,2, \ldots, N$,

$$
\frac{d p_{j}}{d t}=-v\left[\ln p_{j}+a_{L}(\mathbf{p})+b_{L}(\mathbf{p}) e_{j}\right],
$$

where

$$
\begin{aligned}
a_{L}(\mathbf{p}) & =\frac{\sum_{i} e_{i} \sum_{j} e_{j} \ln p_{j}-\sum_{i} \ln p_{i} \sum_{j} e_{j}^{2}}{N \sum_{i} e_{i}^{2}-\left(\sum_{i} e_{i}\right)^{2}}, \\
b_{L}(\mathbf{p}) & =\frac{\sum_{i} \ln p_{i} \sum_{j} e_{j}-\sum_{i} e_{i} \ln p_{i}}{N \sum_{i} e_{i}^{2}-\left(\sum_{i} e_{i}\right)^{2}} .
\end{aligned}
$$

This equation does have the capability of continuously rearranging the distribution so that the perturbed distribution evolves towards the maximal entropy target distribution given by Eq. (2) with energy $\widetilde{E}$. However, in the far-nonequilibrium region it has the defect to imply the unphysical feature that an initially unpopulated eigenstate gets populated at an infinite rate. This feature is in contrast with a wealth of successful models of physical systems in which by limiting our attention to a subset of relevant single-particle eigenstates we get good results, that are relatively robust with respect to adding to the model other less relevant, unpopulated or little populated eigenstates. According to Eq. 4, instead, distributions where some eigenstates are very little populated would survive only for extremely short times.

The equation of motion that we propose for the time evolution of the perturbed distribution is, for $j=$ $1,2, \ldots, N$

$$
\frac{d p_{j}}{d t}=-\frac{1}{\tau}\left[p_{j} \ln p_{j}+\alpha(\mathbf{p}) p_{j}+\beta(\mathbf{p}) e_{j} p_{j}\right],
$$

where

$$
\begin{aligned}
\alpha(\mathbf{p}) & =\frac{\sum_{i} e_{i} p_{i} \sum_{j} e_{j} p_{j} \ln p_{j}-\sum_{i} p_{i} \ln p_{i} \sum_{j} e_{j}^{2} p_{j}}{\sum_{i} e_{i}^{2} p_{i}-\left(\sum_{i} e_{i} p_{i}\right)^{2}}, \\
\beta(\mathbf{p}) & =\frac{\sum_{i} p_{i} \ln p_{i} \sum_{j} e_{j} p_{j}-\sum_{i} e_{i} p_{i} \ln p_{i}}{\sum_{i} e_{i}^{2} p_{i}-\left(\sum_{i} e_{i} p_{i}\right)^{2}} .
\end{aligned}
$$

We show in Section II that the apparently slight modification with respect to Eq. (4) not only fixes the cited defect, while maintaining the relevant overall features of conserving energy, normalization, nonnegativity of the probabilities and maintaining the entropy generation rate nonnegative. It also features existence and uniqueness of the solutions of the Cauchy problem for all times, $-\infty<t<+\infty$, and entails a large class of partiallycanonical equilibrium distributions that are unstable, as well as a single conditionally-stable canonical equilibrium distribution for each value of the energy, as required by a well-known statement of the second law of thermodynamics $[15,16]$.

We show in Section II that the structure of Eq. (5) is the same as that of the general nonlinear quantum equation we discuss in a series of papers written over twenty years ago [17-21] in which we develop and propose a nonlinear quantum dynamics in an attempt to unite ordinary quantum mechanics and general equilibrium and nonequilibrium thermodynamics. As acknowledged also in $[2,22]$, the nonlinear quantum dynamical law first proposed by this author [23] does have very intriguing and appealing mathematical features. We must admit however that the physical interpretation, motivation and context of our pioneering scheme is still considered 'adventurous' [24] by most of the physical community, although we would prefer to term it 'revolutionary' in the sense of Kuhn [25]. For this reason, in this paper we do not 
pursue such controversial interpretation, but we wish to emphasize that - leaving aside its interpretation and focusing attention only on its mathematics - our previous work represents to our knowledge the first time that the steepest-entropy-ascent (or maximal-entropy-generation) ansatz has been explicitly formulated and implemented in a general dynamical law capable of describing the relaxation of arbitrary nonequilibrium states towards thermodynamic equilibrium.

In Section III we provide a derivation of Eq. (5) from the assumption that the occupation probability distribution evolves along the steepest-entropy-ascent trajectory in the state space defined in terms not of the $p_{i}$ 's but of their positive square roots $\sqrt{p_{i}}$ 's. In Section IV we derive a fluctuation-dissipation formulation of the equation and in Section $\mathrm{V}$ a variational formulation.

In Section VI we discuss a simplest degenerate case in which the relaxation equation admits an analytical solution, and we compare results with those numerically derived from the natural extension of Eq. (4) to such case. Finally, in Section VII we show some numerical results that illustrate the general features of the proposed nonlinear relaxation equation.

\section{MAIN FEATURES OF THE ASSUMED NONLINEAR RELAXATION EQUATION}

By analogy with the dynamical law introduced in $[17,19,23,26]$, Eq. (5) may be also written as a ratio of determinants in the form

$$
\frac{d p_{j}}{d t}=-\frac{1}{\tau} \frac{\left|\begin{array}{ccc}
p_{j} \ln p_{j} & p_{j} & e_{j} p_{j} \\
\sum p_{i} \ln p_{i} & 1 & \sum e_{i} p_{i} \\
\sum e_{i} p_{i} \ln p_{i} & \sum e_{i} p_{i} & \sum e_{i}^{2} p_{i}
\end{array}\right|}{\left|\begin{array}{cc}
1 & \sum e_{i} p_{i} \\
\sum e_{i} p_{i} & \sum e_{i}^{2} p_{i}
\end{array}\right|},
$$

where $|\cdot|=\operatorname{det}[\cdot]$, and $\tau$ is assumed constant [27] and may be used to nondimensionalize time (or we may assume $\tau=1$ unit of time).

The resulting rate of entropy generation may be written as a ratio of Gram determinants in the form

$\frac{d S}{d t}=\frac{k_{\mathrm{B}}}{\tau} \frac{\left|\begin{array}{ccc}\sum p_{i}\left(\ln p_{i}\right)^{2} & \sum p_{i} \ln p_{i} & \sum e_{i} p_{i} \ln p_{i} \\ \sum p_{i} \ln p_{i} & 1 & \sum e_{i} p_{i} \\ \sum e_{i} p_{i} \ln p_{i} & \sum e_{i} p_{i} & \sum e_{i}^{2} p_{i}\end{array}\right|}{\left|\begin{array}{cc}1 & \sum e_{i} p_{i} \\ \sum e_{i} p_{i} & \sum e_{i}^{2} p_{i}\end{array}\right|} \geq 0$, where the non-negativity follows from the well-known properties of Gram determinants (see also Section III).

Eq. (5) or the equivalent Eq. (6) is well-behaved in the sense that the following general features can be readily verified (detailed proofs in $[4,17]$ ):

- it conserves the normalization of the distribution and the mean energy $E$ along the entire time evolution;

- it preserves the non-negativity of each $p_{i}$;

- it maintains the rate of entropy generation nonnegative at all times;

- it maintains unoccupied all the initially unoccupied eigenstates; in other words, given a distribution $p_{i}$ and defining the vector $\boldsymbol{\delta}(\mathbf{p})$ of $\delta_{i}$ 's such that, for each $i=1,2, \ldots, N, \delta_{i}=0$ if $p_{i}=0$ or $\delta_{i}=1$ if $p_{i} \neq 0$, the vector $\boldsymbol{\delta}$ is time invariant;

- it drives any arbitrary initial distribution $\mathbf{p}(0)$ towards the partially-canonical (or canonical, if $\delta_{i}=$ 1 for all $p_{i}$ 's) equilibrium distribution, reached as $t \rightarrow \infty$

$$
p_{j}^{\mathrm{pe}}(E, \boldsymbol{\delta})=\frac{\delta_{j} \exp \left(-\beta^{\mathrm{pe}}(E, \boldsymbol{\delta}) e_{j}\right)}{\sum_{i=1}^{N} \delta_{i} \exp \left(-\beta^{\mathrm{pe}}(E, \boldsymbol{\delta}) e_{i}\right)},
$$

where, of course, $\boldsymbol{\delta}=\boldsymbol{\delta}(\mathbf{p}(0))$ and the value of $\beta^{\text {pe }}$ is determined by the initial state through the relation $\sum_{i=1}^{N} e_{i} p_{i}^{\mathrm{pe}}(E, \boldsymbol{\delta})=E=E(\mathbf{p}(0))$. Distributions (8) are those for which $d \mathbf{p} / d t=0$, i.e., that satisfy the equilibrium condition $p_{i} \ln p_{i}=-\alpha p_{i}-\beta e_{i} p_{i}$ for all' $i$ 's and some scalars $\alpha$ and $\beta$.

Moreover, Eq. (6) is well-behaved not only in forward time but also in backward time, consistently with the strongest form of the principle of causality, by which future states of a strictly isolated system should unfold deterministically from initial states along smooth unique trajectories in state domain defined for all times (future as well as past). Indeed, for any given arbitrary 'initial' distribution $\mathbf{p}(0)$ we can follow the unique trajectory $\mathbf{p}(t)$ for $-\infty<t<+\infty$. In forward time the target distributions of all trajectories are given by Eq. $(8), \mathbf{p}(+\infty)=\mathbf{p}^{\mathrm{pe}}(E(\mathbf{p}(0)), \boldsymbol{\delta}(\mathbf{p}(0)))$. The backwardtime earliest (or 'primordial') lowest-entropy distribution $\mathbf{p}(-\infty)$ is also uniquely identified by the given initial distribution $\mathbf{p}(0)$ through Eq. (6), but it is harder to characterize analytically in general. Depending on the given $\mathbf{p}(0)$, the redistribution among energy eigenstates may affect some of the occupation numbers in a non-monotonic way. In the limit as $t \rightarrow-\infty$, however, all $d p_{j} / d t$ 's [the rhs of each of Eqs. (6)] become sign-definite; for example, they may become all positive except for a particular one which tends to $p_{\bar{k}}(-\infty)=1$, so that all others tend to zero, $p_{j \neq \bar{k}}(-\infty)=0$ [this can happen only if the mean energy $E(\mathbf{p}(0))$ is exactly equal to the $\bar{k}$-th energy level, 
i.e., only if $E(\mathbf{p}(0))=e_{\bar{k}}$ ], or they may all tend to zero except for two particular $p_{j}$ 's, say $p_{\bar{j}}$ and $p_{\bar{k}}$ which tend to finite values, clearly with $p_{\bar{j}}+p_{\bar{k}}=1$ (examples in Section VII).

Because the model equation maintains the rate of entropy generation non-negative, the entropy functional $S$ [Eq. (1)] is an $S$-function [16] and, therefore, every thermal-like canonical equilibrium distribution (8) is $\boldsymbol{\delta}$ $E$-conditionally stable, that is, stable with respect to perturbations that do not alter the mean value $E$ of the energy and the set of unoccupied energy eigenstates (described by the zeroes in vector $\boldsymbol{\delta}$ ). These distributions constitute the 'target' highest-entropy states compatible with the mean value of the energy and the invariant subset of unoccupied eigenstates. These distributions, however, are not $E$-conditionally stable, that is, stable with respect to all perturbations that do not alter the mean value $E$. Indeed, starting from a distribution (8), a perturbation that changes a zero probability to an infinitesimal value, makes the perturbed distribution proceed in time by amplifying that probability until a new, different and higher-entropy, target canonical distribution is reached. For a given mean energy $E$, the only canonical distribution that is $E$-conditionally stable is the one for which all energy eigenstates are occupied, i.e., the maximal-entropy canonical distribution (2).

By interpreting the entropy $S$ as a measure of how 'well' the energy is distributed among the available energy eigenstates, the proposed nonlinear dynamics describes a spontaneous internal redistribution of the energy along the path of maximal entropy increase leading towards an 'optimally' distributed (highest-entropy) state compatible with the condition of maintaining unoccupied the initially unoccupied energy eigenstates.

\section{CONSTRUCTION OF THE EQUATION FROM THE STEEPEST-ENTROPY-ASCENT ANSATZ}

In this section, we provide a brief derivation of Eq. (6) from the assumption that the occupation probability distribution evolves along the steepest-entropy-ascent trajectory in the proper state space. We also discuss an important degenerate case.

For the purpose of this derivation, instead of working with the vector $\mathbf{p}$ of the occupation probabilities, we work in terms of their positive square roots [28], $y_{i}=\sqrt{p_{i}}$ and the corresponding vector $\mathbf{y}$. We rewrite the mean energy, normalization and entropy functionals as

$$
\begin{aligned}
& E(\mathbf{y})=\sum_{i=1}^{N} e_{i} y_{i}^{2}, \quad U(\mathbf{y})=\sum_{i=1}^{N} y_{i}^{2}, \\
& S(\mathbf{y})=-k_{\mathrm{B}} \sum_{i=1}^{N} y_{i}^{2} \ln y_{i}^{2}
\end{aligned}
$$

where, of course, $U(\mathbf{y})=1$ for any $\mathbf{y}$. The $i$-th component of the gradients of these functionals are, respectively,

$$
e_{i}^{\prime}=2 e_{i} y_{i}, \quad u_{i}^{\prime}=2 y_{i}, \quad s_{i}^{\prime}=-2 k_{\mathrm{B}}\left(y_{i} \ln y_{i}^{2}+y_{i}\right)
$$

and, therefore, the time-rate-of-change functionals may be written as

$$
\begin{aligned}
\dot{E} & =\left(\dot{\mathbf{y}}, \mathbf{e}^{\prime}\right), \quad \dot{U}=2(\dot{\mathbf{y}}, \mathbf{y}), \\
\dot{S} & =\left(\dot{\mathbf{y}}, \mathbf{s}^{\prime}\right),
\end{aligned}
$$

where $(\cdot, \cdot)$ denotes the scalar product of two vectors [e.g., the normalization condition $U(\mathbf{y})=1$ may be rewritten as $(\mathbf{y}, \mathbf{y})=1]$, and the energy and entropy gradient vectors $\mathbf{e}^{\prime}$ and $\mathbf{s}^{\prime}$ are defined by the components in (10), while we choose to substitute immediately the obvious relation $\mathbf{u}^{\prime}=2 \mathbf{y}$.

In order to maintain $(\dot{\mathbf{y}}, \mathbf{y})=0$ and $\left(\dot{\mathbf{y}}, \mathbf{e}^{\prime}\right)=0$ the vector $\dot{\mathbf{y}}$ must be orthogonal to the linear manifold spanned by $\mathbf{y}$ and $\mathbf{e}^{\prime}$.

For unconstrained maximal entropy generation, $\dot{\mathbf{y}}$ would be in the direction of the gradient $\mathbf{s}^{\prime}$ of the entropy functional $S(\mathbf{y})$; in such case, however, because $\mathbf{s}^{\prime}$ is almost never orthogonal to the $\mathbf{y e}^{\prime}$ manifold, in general $U(\mathbf{y})$ and $E(\mathbf{y})$ would not remain time invariant. Instead, we assume constrained - constant $E(\mathbf{y})$ and $U(\mathbf{y})$ - maximal entropy generation. We obtain it by taking $\dot{\mathbf{y}}$ in the direction of the component of $\mathbf{s}^{\prime}$ orthogonal to the $\mathbf{y e}^{\prime}$ manifold. Denoting such component by $\mathbf{s}_{\perp \mathbf{y} \mathbf{e}^{\prime}}$ we therefore assume

$$
\dot{\mathbf{y}}=\frac{1}{4 k_{\mathrm{B}} \tau(\mathbf{y})} \mathbf{s}_{\perp \mathbf{y e}^{\prime}},
$$

where $\tau(\mathbf{y})$ may be any positive definite functional of $\mathbf{y}$ with dimensions of time, that determines the time rate at which $\mathbf{y}$ evolves along the path of constrained steepest entropy ascent. For simplicity, and for the purpose of comparison with [1], we assume $\tau$ a positive constant as done in our first proposal of this equation of motion in $[17,20,21,23]$.

Using the well-known theory of Gram determinants, we can write an explicit expression for $\mathbf{s}^{\prime}{ }_{\perp \mathbf{y}} \mathbf{e}^{\prime}$. If $\mathbf{y}$ and $\mathbf{e}^{\prime}$ are linearly independent, we have

$$
\mathbf{s}_{\perp \mathbf{y} \mathbf{e}^{\prime}}^{\prime}=\frac{\left|\begin{array}{ccc}
\mathbf{s}^{\prime} & \mathbf{y} & \mathbf{e}^{\prime} \\
\left(\mathbf{s}^{\prime}, \mathbf{y}\right) & (\mathbf{y}, \mathbf{y}) & \left(\mathbf{e}^{\prime}, \mathbf{y}\right) \\
\left(\mathbf{s}^{\prime}, \mathbf{e}^{\prime}\right) & \left(\mathbf{y}, \mathbf{e}^{\prime}\right) & \left(\mathbf{e}^{\prime}, \mathbf{e}^{\prime}\right)
\end{array}\right|}{\left|\begin{array}{ll}
(\mathbf{y}, \mathbf{y}) & \left(\mathbf{e}^{\prime}, \mathbf{y}\right) \\
\left(\mathbf{y}, \mathbf{e}^{\prime}\right) & \left(\mathbf{e}^{\prime}, \mathbf{e}^{\prime}\right)
\end{array}\right|} .
$$

If instead $\mathbf{y}$ and $\mathbf{e}^{\prime}$ are linearly dependent, i.e., if $\mathbf{e}^{\prime}=2 e \mathbf{y}$ for some scalar $e$, the expression is 


$$
\mathbf{s}_{\perp \mathbf{y e}^{\prime}}=\left|\begin{array}{cc}
\mathbf{s}^{\prime} & \mathbf{y} \\
\left(\mathbf{s}^{\prime}, \mathbf{y}\right) & (\mathbf{y}, \mathbf{y})
\end{array}\right| /(\mathbf{y}, \mathbf{y})=\mathbf{s}^{\prime}-\left(\mathbf{s}^{\prime}, \mathbf{y}\right) \mathbf{y}
$$

where we use $(\mathbf{y}, \mathbf{y})=1$. In either case, we readily verify that

$$
\begin{aligned}
\dot{E} & =\left(\dot{\mathbf{y}}, \mathbf{e}^{\prime}\right)=0, \quad \dot{U}=2(\dot{\mathbf{y}}, \mathbf{y})=0, \\
\dot{S} & =\left(\dot{\mathbf{y}}, \mathbf{s}^{\prime}\right)=4 \tau k_{\mathrm{B}}(\dot{\mathbf{y}}, \dot{\mathbf{y}}),
\end{aligned}
$$

from which we see that the rate of entropy generation is related to the norm of $\dot{\mathbf{y}}$ and is positive definite.

Combining Eqs. (12) and (13a) we find

$$
4 k_{\mathrm{B}} \tau \dot{\mathbf{y}}=\mathbf{s}^{\prime}-a(\mathbf{y}) \mathbf{y}-b(\mathbf{y}) \mathbf{e}^{\prime},
$$

where

$$
\begin{aligned}
& a(\mathbf{y})=\frac{\left(\mathbf{s}^{\prime}, \mathbf{y}\right)\left(\mathbf{e}^{\prime}, \mathbf{e}^{\prime}\right)-\left(\mathbf{s}^{\prime}, \mathbf{e}^{\prime}\right)\left(\mathbf{e}^{\prime}, \mathbf{y}\right)}{(\mathbf{y}, \mathbf{y})\left(\mathbf{e}^{\prime}, \mathbf{e}^{\prime}\right)-\left(\mathbf{y}, \mathbf{e}^{\prime}\right)\left(\mathbf{e}^{\prime}, \mathbf{y}\right)}, \\
& b(\mathbf{y})=\frac{\left(\mathbf{s}^{\prime}, \mathbf{e}^{\prime}\right)(\mathbf{y}, \mathbf{y})-\left(\mathbf{s}^{\prime}, \mathbf{y}\right)\left(\mathbf{y}, \mathbf{e}^{\prime}\right)}{(\mathbf{y}, \mathbf{y})\left(\mathbf{e}^{\prime}, \mathbf{e}^{\prime}\right)-\left(\mathbf{y}, \mathbf{e}^{\prime}\right)\left(\mathbf{e}^{\prime}, \mathbf{y}\right)}
\end{aligned}
$$

and, setting back $p_{i}=y_{i}^{2}$ and $\dot{p}_{i}=2 y_{i} \dot{y}_{i}$ we readily obtain Eq. (5) and the identities $\alpha(\mathbf{p})=1+a(\mathbf{y}) / 2 k_{\mathrm{B}}$ and $\beta(\mathbf{p})=b(\mathbf{y}) / k_{\mathrm{B}}$.

Similarly, combining Eqs. (12) and (13b) we find

$$
4 k_{\mathrm{B}} \tau \dot{\mathbf{y}}=\mathbf{s}^{\prime}-\left(\mathbf{s}^{\prime}, \mathbf{y}\right) \mathbf{y}
$$

and, therefore, in the degenerate case of $\mathbf{y}$ and $\mathbf{e}^{\prime}$ linearly dependent, the relaxation equations are, for $j=$ $1,2, \ldots, N$

$$
\frac{d p_{j}}{d t}=-\frac{1}{\tau}\left[p_{j} \ln p_{j}-p_{j}\left(\sum_{i} p_{i} \ln p_{i}\right)\right],
$$

or, equivalently,

$$
\frac{d p_{j}}{d t}=-\frac{1}{\tau}\left|\begin{array}{cc}
p_{j} \ln p_{j} & p_{j} \\
\sum p_{i} \ln p_{i} & 1
\end{array}\right| .
$$

In this case, the rate of entropy generation may be written as

$$
\frac{d S}{d t}=\frac{k_{\mathrm{B}}}{\tau}\left|\begin{array}{cc}
\sum p_{i}\left(\ln p_{i}\right)^{2} & \sum p_{i} \ln p_{i} \\
\sum p_{i} \ln p_{i} & 1
\end{array}\right| \geq 0,
$$

where the nonegativity follows from the well-known properties of Gram determinants.

Equation (16c) substitutes (6) when $\mathbf{e}^{\prime}=2 e \mathbf{y}$ for some scalar $e$ or, equivalently, when $e_{i} p_{i}=e p_{i}$ for every $i$, that is, when the populated eigenstates all correspond to the same energy level. If satisfied at one instant in time this condition is satisfied at all times, both forward and backward in time. It follows that in such degenerate cases the entire time evolution is governed by Eq. (16b).
In the general nondegenerate cases, i.e., when at one time (and, hence, at all times) $e_{i} p_{i} \neq E p_{i}$ for two or more $i$ 's, where $E$ is the mean energy, the time evolution is entirely governed by Eq. (5) [or, equivalently, (6) or (15)]. This also implies that the denominators of $\alpha(\mathbf{p})$ and $\beta$ (p) [or, equivalently, of $a(\mathbf{y})$ and $b(\mathbf{y})$ ] remain positive definite at all times and, hence, the entire time evolution is welldefined.

As stated above, the feature that unpopulated eigenstates remain unpopulated is extremely important as it is compatible, for example, with the widely accepted and successful possibility to describe real systems by means of simplified models with a limited number of relevant energy eigenstates.

The same feature does not hold for equation (4), because it implies that an initially unpopulated eigenstate gets populated at an infinite rate. For the same reason, Eq. (4) does not allow tracing the time evolution backward in time beyond the instant when the first eigenstate becomes unpopulated, for at earlier times the condition $p_{i} \geq 0$ is not satisfied.

\section{FLUCTUATION-DISSIPATION FORMULATION}

It is noteworthy that Eq. (6) admits a general fluctuation-dissipation formulation and interpretation. To see this, we introduce the energy and entropy fluctuation functionals as follows

$$
\begin{aligned}
\langle\Delta E \Delta E\rangle(\mathbf{p}) & =\sum_{i} p_{i}\left[e_{i}-E(\mathbf{p})\right]^{2} \\
& =\sum_{i} p_{i} e_{i}^{2}-\left(\sum_{i} p_{i} e_{i}\right)^{2} \\
& =\frac{1}{4}\left|\begin{array}{cc}
(\mathbf{y}, \mathbf{y}) & \left(\mathbf{e}^{\prime}, \mathbf{y}\right) \\
\left(\mathbf{y}, \mathbf{e}^{\prime}\right) & \left(\mathbf{e}^{\prime}, \mathbf{e}^{\prime}\right)
\end{array}\right|, \\
\langle\Delta S \Delta S\rangle(\mathbf{p}) & =\sum_{i} p_{i}\left[-k_{\mathrm{B}} \ln p_{i}-S(\mathbf{p})\right]^{2} \\
& =k_{\mathrm{B}}^{2} \sum_{i} p_{i}\left(\ln p_{i}\right)^{2}-k_{\mathrm{B}}^{2}\left(\sum_{i} p_{i} \ln p_{i}\right)^{2}, \\
\langle\Delta E \Delta S\rangle(\mathbf{p}) & =\sum_{i} p_{i}\left[e_{i}-E(\mathbf{p})\right]\left[-k_{\mathrm{B}} \ln p_{i}-S(\mathbf{p})\right],
\end{aligned}
$$

and rewrite functionals $\beta(\mathbf{p})$ and $\alpha(\mathbf{p})$ as

$$
\begin{aligned}
& \beta(\mathbf{p})=\frac{1}{k_{\mathrm{B}}} \frac{\langle\Delta E \Delta S\rangle(\mathbf{p})}{\langle\Delta E \Delta E\rangle(\mathbf{p})}, \\
& \alpha(\mathbf{p})=\frac{S(\mathbf{p})}{k_{\mathrm{B}}}-\beta(\mathbf{p}) E(\mathbf{p}) .
\end{aligned}
$$

At the thermodynamic equilibrium distribution with energy $E, \mathbf{p}^{\mathrm{se}}(E)$, we have

$$
\begin{aligned}
& k_{\mathrm{B}} \beta\left(\mathbf{p}^{\mathrm{se}}(E)\right)=k_{\mathrm{B}} \beta^{\mathrm{se}}(E)=1 / T(E), \\
& k_{\mathrm{B}} \alpha\left(\mathbf{p}^{\mathrm{se}}(E)\right)=k_{\mathrm{B}} \alpha^{\mathrm{se}}(E)=S^{\mathrm{se}}(E)-E / T(E),
\end{aligned}
$$

and in Eq. (24) we recognize the thermodynamicequilibrium Massieu characteristic function [15] 


$$
M^{\mathrm{se}}=S-E / T .
$$

It is therefore natural, in this framework, to adopt the following generalization of the Massieu function to arbitrary nonequilibrium distributions

$$
M(\mathbf{p})=k_{\mathrm{B}} \alpha(\mathbf{p})=S(\mathbf{p})-k_{\mathrm{B}} \beta(\mathbf{p}) E(\mathbf{p}),
$$

with $\beta(\mathbf{p})$ given by Eq. (21). The corresponding fluctuations functional is

$$
\langle\Delta M \Delta M\rangle(\mathbf{p})=\sum_{i} p_{i}\left[-k_{\mathrm{B}} \ln p_{i}-k_{\mathrm{B}} \beta(\mathbf{p}) e_{i}-M(\mathbf{p})\right]^{2} .
$$

We can readily verify that [Eq. (7)] may be rewritten as

$$
\frac{d S}{d t}=\frac{1}{k_{\mathrm{B}} \tau}\langle\Delta M \Delta M\rangle
$$

and, therefore, the rate of entropy generation is directly proportional to the fluctuations of our generalized Massieu function [29]. Such fluctuations are related to entropy and energy fluctuations through functional $k_{\mathrm{B}} \beta(\mathbf{p})$ as follows

$$
\langle\Delta M \Delta M\rangle(\mathbf{p})=\langle\Delta S \Delta S\rangle(\mathbf{p})-k_{\mathrm{B}}^{2} \beta(\mathbf{p})^{2}\langle\Delta E \Delta E\rangle(\mathbf{p}),
$$

and become zero at every canonical thermodynamicequilibrium distribution $\mathbf{p}^{\mathrm{se}}(E)$, Eq. (2), and at every partially-canonical equilibrium distribution $\mathrm{p}^{\mathrm{pe}}(E, \boldsymbol{\delta})$, Eq. (8), as well.

It is noteworthy that the functional $k_{\mathrm{B}} \beta(\mathbf{p})$, which is well-defined by Eq. (21) only for distributions with $\langle\Delta E \Delta E\rangle \neq 0$, may be interpreted in this framework as a natural generalization to nonequilibrium of the inverse temperature, at least inasfar as for $t \rightarrow+\infty$ it tends to the thermodynamic-equilibrium inverse temperature $k_{\mathrm{B}} \beta^{\text {se }}$ of distribution (2) or the partial equilibrium inverse temperature $k_{\mathrm{B}} \beta^{\text {pe }}$ of distribution (8).

The special case of distributions with $\langle\Delta E \Delta E\rangle=0$ happens if and only if $\mathbf{e}^{\prime}=2 e \mathbf{y}$ for some scalar $e$ (see Section III). In such special degenerate case, $\langle\Delta E \Delta E\rangle$ remains zero along the entire time evolution, which is given by Eq. (16b), and the role of the Massieu function is taken up by the entropy $S$, for both equilibrium and nonequilibrium distributions. The fluctuation-dissipation form of the rate of entropy generation [Eq. (17)] becomes the following

$$
\frac{d S}{d t}=\frac{1}{k_{\mathrm{B}} \tau}\langle\Delta S \Delta S\rangle,
$$

and in this special degenerate case the canonical and partially-canonical equilibrium distributions all have $\langle\Delta S \Delta S\rangle=0$, for they consist of $N_{\boldsymbol{\delta}}=(\boldsymbol{\delta}, \boldsymbol{\delta})$ probabilities $p_{i}$ all equal to $1 / N_{\delta}$ and of $N-N_{\delta}$ all equal to zero.
As regards the fluctuation-dissipation relations, the various well formulated arguments, derivations and interpretations discussed for Eq. (4) by Englman in the Appendix of Ref. [1] and based on the steepest-entropyascent ansatz - first introduced in quantum thermodynamics by the present author [19] - apply with minor modifications also for our better-behaved dynamical equation Eq. (5). In addition, we prove in [26] that Eq. (5) implies a generalized Onsager reciprocity theorem.

\section{VARIATIONAL FORMULATION}

In terms of the $y_{i}=\sqrt{p_{i}}$ notation, we can derive our equation of motion also as a result of the following equivalent variational formulation (along the lines recently proposed in [4])

$$
\begin{array}{r}
\max _{\dot{\mathbf{y}}} \dot{S}=\left(\dot{\mathbf{y}}, \mathbf{s}^{\prime}\right) \quad \text { subject to } \dot{E}=\left(\dot{\mathbf{y}}, \mathbf{e}^{\prime}\right)=0, \\
\dot{U}=(\dot{\mathbf{y}}, \mathbf{y})=0, \text { and }(\dot{\mathbf{y}}, \dot{\mathbf{y}})=\xi(\mathbf{y}),
\end{array}
$$

where the last constraint implies that we maximize the entropy generation rate only with respect to the 'direction' of $\dot{\mathbf{y}}$, i.e., at every given $\mathbf{y}$ we select the maximizing $\dot{\mathbf{y}}$ among a subset of vectors that share the same (but otherwise arbitrary) norm $\xi(\mathbf{y})$. For $\mathbf{y}$ and $\mathbf{e}^{\prime}$ linearly independent, using the standard method, we associate the Lagrange multipliers $a, b$ and $4 k_{\mathrm{B}} \tau$ with the constraints, and from Eq. (14) and the necessary Euler-Lagrange conditions

$$
\frac{\partial}{\partial \dot{\mathbf{y}}}\left[\left(\dot{\mathbf{y}}, \mathbf{s}^{\prime}\right)-a(\dot{\mathbf{y}}, \mathbf{y})-b\left(\dot{\mathbf{y}}, \mathbf{e}^{\prime}\right)-4 k_{\mathrm{B}} \tau(\dot{\mathbf{y}}, \dot{\mathbf{y}})\right]=0,
$$

we readily obtain Eq. (15) as well as, upon substitution into the constraints, the multipliers given by Eqs. (15b) and $(15 \mathrm{c})$, and the square norm of $\dot{\mathbf{y}}$,

$$
\begin{aligned}
\xi(\mathbf{y}) & =\frac{\dot{S}}{4 k_{\mathrm{B}} \tau}=\frac{1}{16 k_{\mathrm{B}}^{2} \tau^{2}} \frac{\Gamma\left(\mathbf{s}^{\prime}, \mathbf{y}, \mathbf{e}^{\prime}\right)}{\Gamma\left(\mathbf{y}, \mathbf{e}^{\prime}\right)} \\
& =\frac{1}{16 k_{\mathrm{B}}^{2} \tau^{2}} \frac{\left|\begin{array}{lll}
\left(\mathbf{s}^{\prime}, \mathbf{s}^{\prime}\right) & \left(\mathbf{y}, \mathbf{s}^{\prime}\right) & \left(\mathbf{e}^{\prime}, \mathbf{s}^{\prime}\right) \\
\left(\mathbf{s}^{\prime}, \mathbf{y}\right) & (\mathbf{y}, \mathbf{y}) & \left(\mathbf{e}^{\prime}, \mathbf{y}\right) \\
\left(\mathbf{s}^{\prime}, \mathbf{e}^{\prime}\right) & \left(\mathbf{y}, \mathbf{e}^{\prime}\right) & \left(\mathbf{e}^{\prime}, \mathbf{e}^{\prime}\right)
\end{array}\right|}{\left|\begin{array}{ll}
(\mathbf{y}, \mathbf{y}) & \left(\mathbf{e}^{\prime}, \mathbf{y}\right) \\
\left(\mathbf{y}, \mathbf{e}^{\prime}\right) & \left(\mathbf{e}^{\prime}, \mathbf{e}^{\prime}\right)
\end{array}\right|},
\end{aligned}
$$

where $\Gamma$ denotes the Gram determinant of the argument vectors.

Similarly, for the degenerate cases with $\mathbf{y}$ and $\mathbf{e}^{\prime}$ linearly dependent, the normalization and constant energy conditions collapse into a unique constraint with which we associate the Lagrange multiplier $c$, and by the same standard procedure we obtain Eq. (16a) and, upon substitution into the constraints, the multiplier $c=\left(\mathbf{s}^{\prime}, \mathbf{y}\right)$, and the square norm of $\dot{\mathbf{y}}$, 


$$
\begin{aligned}
\xi(\mathbf{y}) & =\frac{\dot{S}}{4 k_{\mathrm{B}} \tau}=\frac{1}{16 k_{\mathrm{B}}^{2} \tau^{2}} \Gamma\left(\mathbf{s}^{\prime}, \mathbf{y}\right) \\
& =\frac{1}{16 k_{\mathrm{B}}^{2} \tau^{2}}\left|\begin{array}{cc}
\left(\mathbf{s}^{\prime}, \mathbf{s}^{\prime}\right) & \left(\mathbf{y}, \mathbf{s}^{\prime}\right) \\
\left(\mathbf{s}^{\prime}, \mathbf{y}\right) & (\mathbf{y}, \mathbf{y})
\end{array}\right| .
\end{aligned}
$$

\section{SIMPLEST CASE: TWO-LEVEL PARTICLES WITH DEGENERATE EIGENSTATES}

The simplest mathematical form of the model equation that derives from the equation of motion proposed in the previous sections, is obtained when we have an isolated, closed gas composed of non-interacting identical two-level particles with degenerate energy levels, such as electonic spins in the absence of an applied magnetic field. Then $N=2$, both levels have energy $e_{1}=e_{2}=e$, the occupation probabilities of the two corresponding eigenstates are $p_{1}=1-p$ and $p_{2}=p$, respectively, and the model equation (16c) for redistribution among the two eigenstates becomes

$$
\frac{d p}{d t}=p(1-p) \ln \frac{1-p}{p}
$$

where we set $\tau=1$. The rate of entropy generation $\left(k_{\mathrm{B}}=1\right)$ is

$$
\dot{S}=p(1-p)\left(\ln \frac{1-p}{p}\right)^{2}
$$

Not only equation (35) is well-behaved at all times (existence and uniqueness of the solution for any initial $p(0)$ with $0 \leq p(0) \leq 1$ ), but it can also be integrated to yield

$$
t=\int_{p(0)}^{p(t)} \frac{d p}{p(1-p) \ln \frac{1-p}{p}}=\ln \frac{\ln \frac{1-p(0)}{p(0)}}{\ln \frac{1-p(t)}{p(t)}}
$$

or, equivalently,

$$
\begin{aligned}
p(t) & =\frac{1}{1+\left(\frac{1-p(0)}{p(0)}\right)^{\exp (-t / \tau)}} \\
& =\frac{1}{2}+\frac{1}{2} \tanh \left(-\frac{1}{2} \exp (-t / \tau) \ln \frac{1-p(0)}{p(0)}\right),
\end{aligned}
$$

from which we readily find $p(\infty)=1 / 2$ and

$$
p(-\infty)=\frac{1}{1+\left(\frac{1-p(0)}{p(0)}\right)}=\left\{\begin{array}{ll}
0 \text { for } p(0)<1 / 2 \\
1 \text { for } p(0)>1 / 2
\end{array} .\right.
$$

By analogy, the extension to this degenerate case of the model equation proposed in [1] is $d p_{j} / d t=v\left(-\ln p_{j}+a_{L}\right)$ with $2 a_{L}=\sum_{i=1}^{2} \ln p_{i}=\ln p+\ln (1-p)$ that is (setting $v=1 / 2$ and adding, for clarity, the subscript $L$ )

$$
\frac{d p_{L}}{d t}=\frac{1}{4} \ln \frac{1-p_{L}}{p_{L}},
$$

which yields the entropy generation rate

$$
\dot{S}_{L}=\frac{1}{4}\left(\ln \frac{1-p_{L}}{p_{L}}\right)^{2} .
$$

Figure 1 shows a comparison between the time dependence (38) implied by our rate equation (35) and that obtained by numerical solution (by a standard Runge-Kutta method) of the rate equation (40), for both $p(-\infty)=0=$ $p_{L}(0)$ and $p(-\infty)=1=p_{L}(0)$. For the purpose of comparison, time $t=0$ is selected where $p_{L}(0)=0$ or 1 and the initial state $p(0)$ is selected so as to emphasize that in the limit as $t \rightarrow+\infty$ we have $p(t) \approx p_{L}(t)$. In fact, we readily verify from both Eqs. (35) and (40) that the two time dependences have the same asymptotic behavior as they approach the equilibrium distribution, that is,

$$
\frac{d p}{d t} \approx \frac{1}{2}-p \quad \text { and } \quad \dot{S} \approx 4\left(\frac{1}{2}-p\right)^{2} .
$$

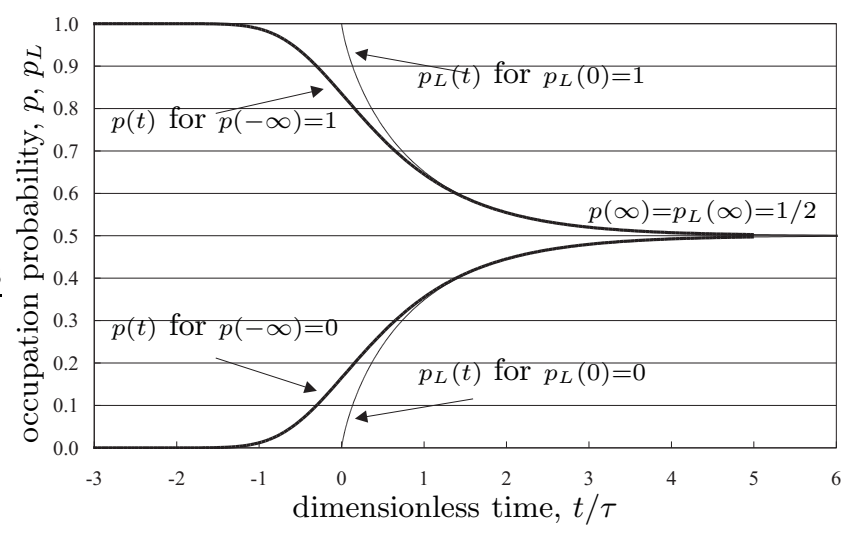

FIG. 1. Comparison of the time dependences $p(t)$ and $p_{L}(t)$ respectively implied by the rate equation (35) that we propose and the rate equation (40) discussed in [1].

This simplest case brings out the evident different behavior at early times and the unphysical feature of the solution of Eq. (40) at $p_{L}=0$ where the repopulation rate is infinite, implying that no unpopulated eigenstate can survive unpopulated. Instead, our Eq. (35) maintains unpopulated any initially unpopulated eigenstate, and it also maintains relatively little populated an initially little populated eigenstate for a lapse of time that is quantified by Eq. (37) and depends on how close the 
initial value $p(0)$ is to zero. For example, the time required to take $p(0)=10^{-2 n}$ to $p(t)=10^{-2}$ is $t \approx \ln n$ (that is, $t \approx \tau \ln n$ ).

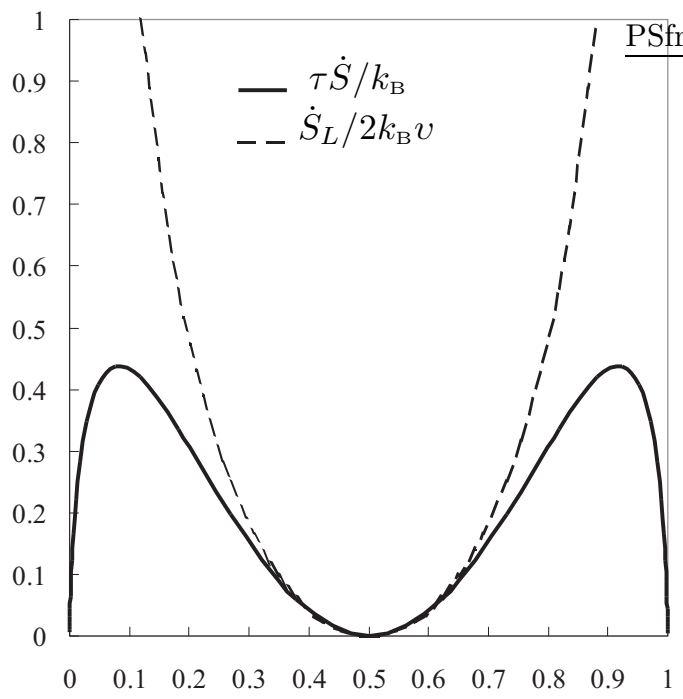

occupation probability, $p, p_{L}$

FIG. 2. Comparison between the entropy generation rate $\dot{S}$ versus $p$ as given by Eq. (36) for $k_{\mathrm{B}}=1$ and $\tau=1$ and $\dot{S}_{L}$ versus $p_{L}$ as given by Eq. (41) for $k_{\mathrm{B}}=1$ and $v=1 / 2$.

Figure 2 shows a plot of the entropy generation rate $\dot{S}$ versus $p$ obtained from Eq. (36) compared with $\dot{S}_{L}$ versus $p_{L}$ as obtained from Eq. (41), where again the essential differences for small values of $p$ and $1-p$ are singled out.

\section{NUMERICAL RESULTS}

The energy versus entropy diagram introduced by Gibbs represents the intersection with the $E-S$ plane of the $E-S-V-\mathbf{n}$ surface representing the stable thermodynamic equilibrium states of a system, assuming that the energy eigenvalues depend on the volume $V$ and the amounts of constituents $\mathbf{n}$, so that the surface is represented by the so-called fundamental relation $S=S\left(E,\left\{e_{j}(V, \mathbf{n})\right\}\right)$. In [15] the use of such diagram has been extended to include the projection onto the $E-S$ plane of all other states, i.e., not only the stable equilibrium states but also the non-equilibrium and the non-stable equilibrium states, with given fixed values of $V$ and $\mathbf{n}$ and, therefore, a given fixed set of energy eigenvalues. On such diagram, therefore, one point represents in general a multitude of distributions, except at every point of maximal entropy for each given value of $E$ ( $V$ and $\mathbf{n}$ are fixed) which corresponds to a unique canonical distribution (2), i.e., a unique stable thermodynamic equilibrium state.
For a four-level nondegenerate system, Figure 3 represents on the diagram the families of possible canonical (2) and partially-canonical (8) equilibrium distributions which in our dynamics are the only ones with zero entropy generation rate. We recall that the slope of these curves is related to the parameter $\beta^{\mathrm{pe}}(E, \boldsymbol{\delta})$ because $\partial S^{\mathrm{pe}}(E, \boldsymbol{\delta}) /\left.\partial E\right|_{\boldsymbol{\delta}}=k_{\mathrm{B}} \beta^{\mathrm{pe}}(E, \boldsymbol{\delta})$, which for the canonical distribution (all $\delta_{i}$ 's equal to unity) is $\partial S(E) / \partial E=$ $k_{\mathrm{B}} \beta(E)=1 / T(E)$.

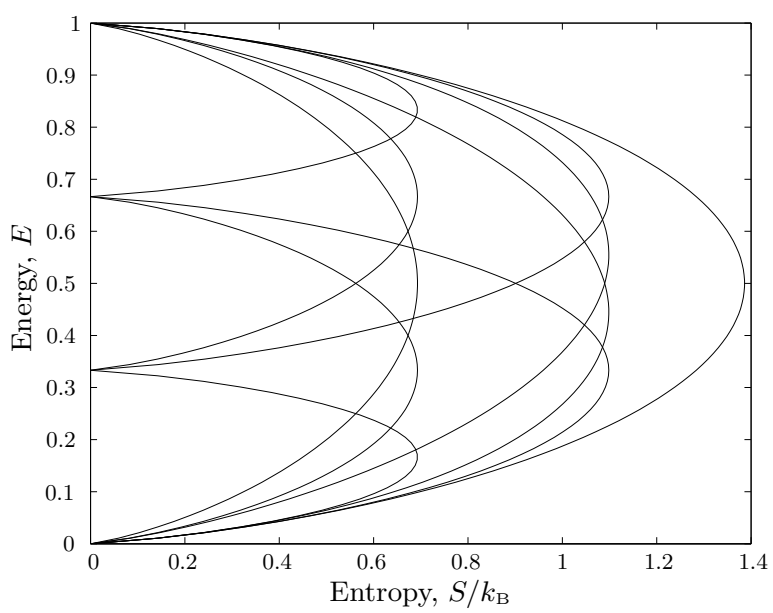

FIG. 3. Representation on an energy versus entropy diagram (for $N=4$ and nondegenerate eigenstates with energies $\mathbf{e}=[0,1 / 3,2 / 3,1])$ of the families of possible canonical and partially-canonical equilibrium distributions which in our dynamics are the only ones with zero entropy generation rate. For example, a horizontal line at $E=0.4$ intersects seven different families of partially canonical states.

The number of possible distributions that share a given pair of values of $E$ and $S$ is in general an $(N-3)$-fold infinity except at maximal entropy for each value of $E$, where the distribution is unique, and at few other notable exceptions such as at minimal entropy for each given $E$ where the distribution may be unique or sometimes many-fold. For all possible distributions represented by a given point on the $E-S$ diagram, we may evaluate the rate of entropy generation $d S / d t$ according to Eq. (6) and select the highest value, that we denote by $\dot{S}_{\max }(E, S)$. The result of this numerical computation is sketched in Figure 4 where the iso- $\dot{S}_{\max }$ contour curves are plotted on the entire allowed domain on the energy versus entropy diagram (of course, under the restriction to the subset of states specified in the Introduction). 


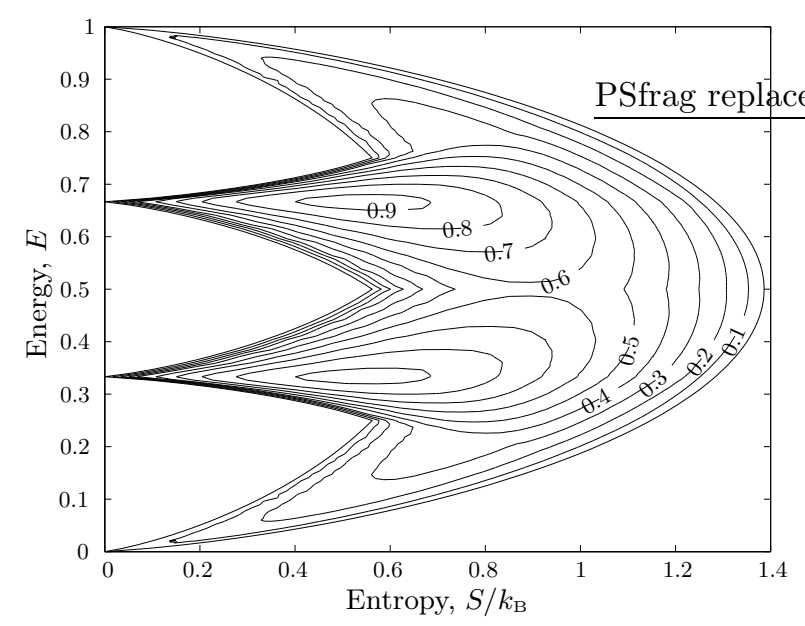

FIG. 4. Representation on an energy versus entropy diagram (for $N=4$ and nondegenerate eigenstates with energies $\mathbf{e}=[0,1 / 3,2 / 3,1])$ of the iso- $\dot{S}_{\max }$ contour curves where $\dot{S}_{\text {max }}$ represents at each point in the diagram the highest value of the rate of entropy generation $d S / d t$ according to Eq. (6) among all the possible distributions represented by that point.

The next Figures show typical time dependences of the occupation probabilities that result from the numerical integration (by means of a standard Runge-Kutta algorithm) of Eq. (6) both in forward and backward time. All trajectories in these Figures refer to a system with $N=4$ and nondegenerate eigenstates with $\mathbf{e}=[0,1 / 3,2 / 3,1]$, and all have the same mean energy $E=2 / 5$; they all tend, of course, to the canonical distribution $\mathbf{p}^{\mathrm{se}}(2 / 5)=[0.3474,0.2722,0.2133,0.1671]$ that has inverse temperature $\beta^{\text {se }}(2 / 5)=0.7321$. They are obtained by assuming for all cases an initial distribution $\mathbf{p}(0)$ obtained by perturbing the canonical distribution $\mathbf{p}^{\text {se }}(E)$ [Eq. (2)] according to Eq. (3) with the energy preserving perturbing factors defined as follows, for $j=1,2, \ldots, N$

$$
f_{j}=1-\lambda+\lambda \frac{p_{j}^{\mathrm{pe}}(E, \boldsymbol{\delta})}{p_{j}^{\mathrm{se}}(E)} \quad \text { with } 0<\lambda<1
$$

where $\lambda$ is otherwise arbitrary, and also $\delta$ is arbitrarily chosen among the possible vectors of 0's and 1's compatible with the given value of $E$ and form (8) of the distribution $\mathbf{p}^{\mathrm{pe}}(E, \boldsymbol{\delta})$ (see Figure 3), where $\beta^{\mathrm{pe}}(E, \boldsymbol{\delta})$ is computed by solving the relation $\sum_{i} p_{i}^{\mathrm{pe}}(E, \boldsymbol{\delta})=E$. For all subsequent Figures we use $\lambda=0.9$.

Figure 5 shows the time dependence of the occupation probabilities that results under the assumptions just cited using $E=2 / 5, \lambda=0.9$ and $\boldsymbol{\delta}=[1,1,0,1]$ in Eq. (43) and subsequently substituting in Eqs. (3), that is,

$$
\mathbf{p}(0)=\lambda \mathbf{p}^{\mathrm{pe}}(E, \boldsymbol{\delta})+(1-\lambda) \mathbf{p}^{\mathrm{se}}(E) .
$$

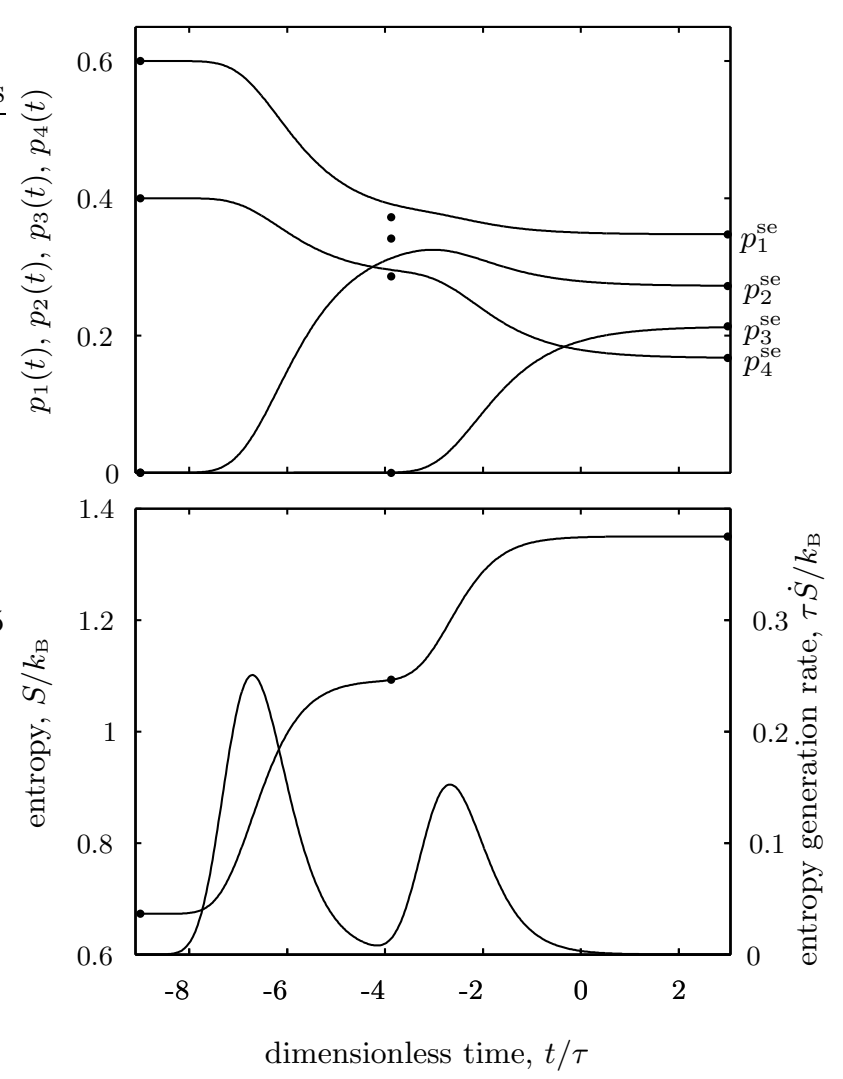

FIG. 5. Top: typical time dependences of the occupation probabilities that result from the numerical integration of Eq. (6) both forward and backward in time, for $N=4$, $\mathbf{e}=[0,1 / 3,2 / 3,1]$, energy $E=2 / 5$, initial state at $t=0$ from Eq. (44) with $\lambda=0.9$ and $\delta=[1,1,0,1]$. The dots on the right represent the maximal entropy distribution; the dots at the left represent the lowest-entropy or 'primordial' distribution; the dots in the middle represent the $\mathbf{p}^{\mathrm{pe}}(E, \boldsymbol{\delta})$ distribution used in Eq. (44) to select the $t=0$ state, plotted at the instant in time when the entropy of the time-varying trajectory is equal to the entropy of the $\mathbf{p}^{\mathrm{pe}}(E, \boldsymbol{\delta})$ distribution. Bottom: the corresponding time dependence of the entropy (left axis) and the entropy generation rate (right axis).

It is noteworthy that when the trajectory gets very close to the partially-canonical unstable-equilibrium distribution $\mathbf{p}^{\mathrm{pe}}(E=2 / 5, \boldsymbol{\delta}=[1,1,0,1])$ the entropy surface presents a local 'plateaux' and the entropy generation rate drops almost to zero, but shortly after the trajectory bends in a direction of steeper slope that drives the generation up again until the canonical distribution $\mathbf{p}^{\mathrm{se}}(E)=[0.3474,0.2722,0.2133,0.1671]$ is finally approached, with inverse temperature $\beta^{\text {se }}(2 / 5)=0.7321$. Of course, the entropy is a monotonically increasing function of time along the entire trajectory.

Figure 6 shows the same trajectory as well as six other trajectories, but instead of plotting the time dependence of the occupation probabilities we plot them against entropy. The initial (time $t=0$ ) distribution 
used to obtain these seven sample trajectories are obtained from Eq. (44) with $E=2 / 5, \lambda=0.9$ and each of the seven partially canonical states corresponding to the given value of the energy. These seven states are easily identified on the $E-S$ diagram in Figure 3 by drawing a horizontal line at $E=0.4$. For the first, third, and sixth trajectories we use the $\mathbf{p}^{\mathrm{pe}}(E, \boldsymbol{\delta})$ states with $\boldsymbol{\delta}=[1,0,1,0], \boldsymbol{\delta}=[1,0,0,1]$ and $\boldsymbol{\delta}=[0,1,0,1]$, respectively, which [as apparent from the subsequent Figure 7] are lowest-entropy boundary points of the entropy surface for the given energy, and turn out to be also the 'primordial' states of the corresponding trajectories. For the remaining trajectories we use the $\mathbf{p}^{\mathrm{pe}}(E, \boldsymbol{\delta})$ states with $\boldsymbol{\delta}=[1,1,1,0], \boldsymbol{\delta}=[1,1,0,1], \boldsymbol{\delta}=[1,0,1,1]$, and $\boldsymbol{\delta}=[0,1,1,1]$, respectively. These too are boundary points of the entropy surface, but they correspond to partial maxima (over the subset of distributions with one unoccupied eigenstate as specified by the corresponding zero element of $\boldsymbol{\delta}$ ). It is seen that these partial maxima affect the trajectories passing nearby by acting as partial attractors especially in the initial phase of the time evolution.

Figure 7 is a more elaborate representation of the same seven trajectories. They are shown four times from different perspectives on the backgorund of contour plots of the entropy surface, for four pairs of occupation probabilities. Indeed, for $N=4$ and fixed energy $E$, the number of independent occupation probabilities is two. Thus for four pairs of probabilities $\left(p_{1}-p_{2}, p_{2}-p_{3}, p_{3}-p_{4}\right.$, $p_{4}-p_{1}$ ), we draw the contour plot of the entropy surface over the entire domain of allowed values (which of course are contained in a triangular region of the first quadrant), and over this plot we draw the seven trajectories (and the seven partially canonical states used to choose them). To save space, we then rotate each of the four graphs (respectively by 45, 135, 225, 315 degrees) and combine them on the same graph in Figure 7. The figure visualizes clearly that the trajectories indeed follow paths of locally-steepest-entropy-ascent and unfold smoothly also backward in time to the 'primordial' states. We also note that these lowest-entropy states exhibit a singular behavior in that, for example, state $[2 / 5,0,3 / 5,0]$ is the primordial state for two entirely different trajectories, state $[3 / 5,0,0,2 / 5]$ for three other, and state $[0,9 / 10,0,1 / 10]$ for the remaining two. Moreover, the partially canonical states appear as partial attractors of trajectories passing nearby, as seen quite clearly for the second, fourth and fifth trajectory of Figure 6, which are partially attracted by the partially canonical states with $\boldsymbol{\delta}=[1,1,1,0], \boldsymbol{\delta}=[1,1,0,1]$ and $\boldsymbol{\delta}=[1,0,1,1]$, respectively.
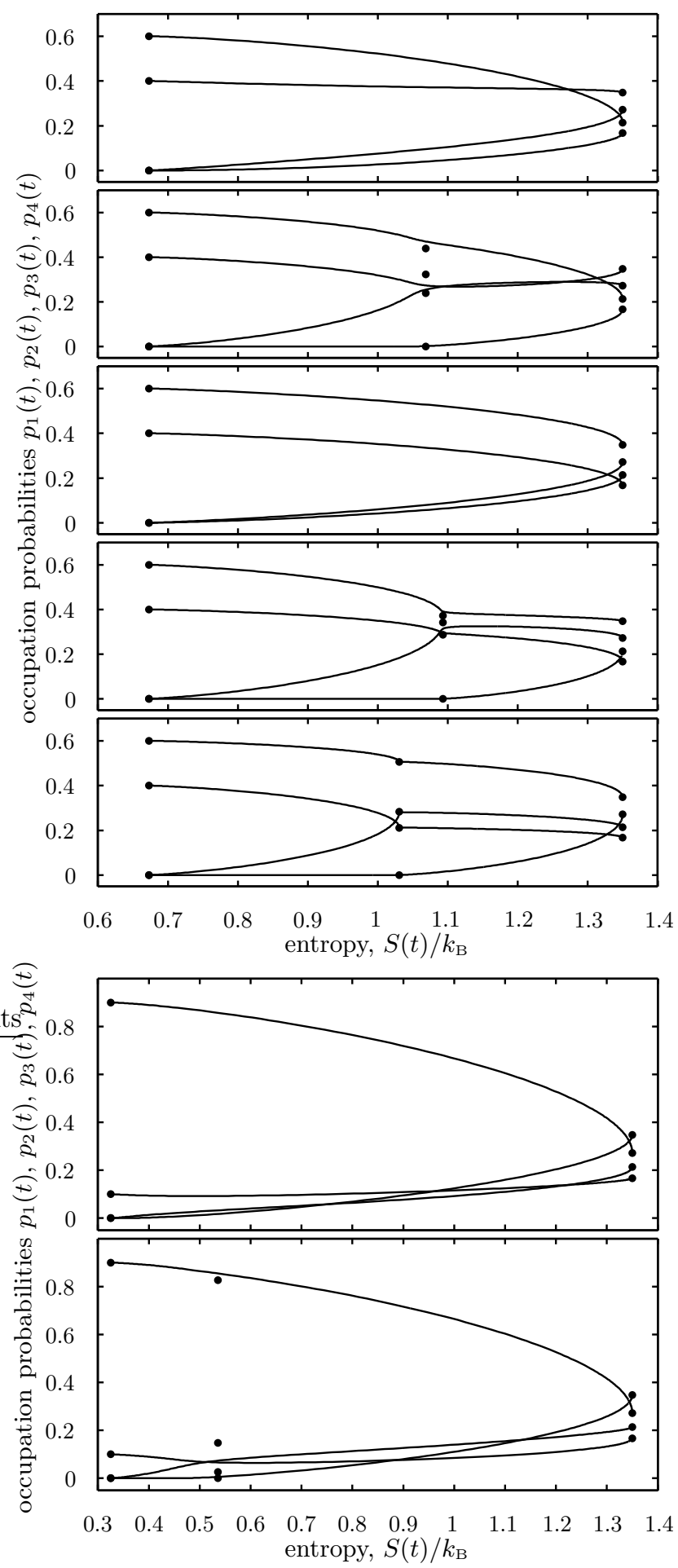

FIG. 6. Plots of $p_{i}(t)$ versus $S(t)$ for seven sample time dependences of the occupation probabilities that result from the numerical integration of Eq. (6) both forward and backward in time, for different initial distributions. 


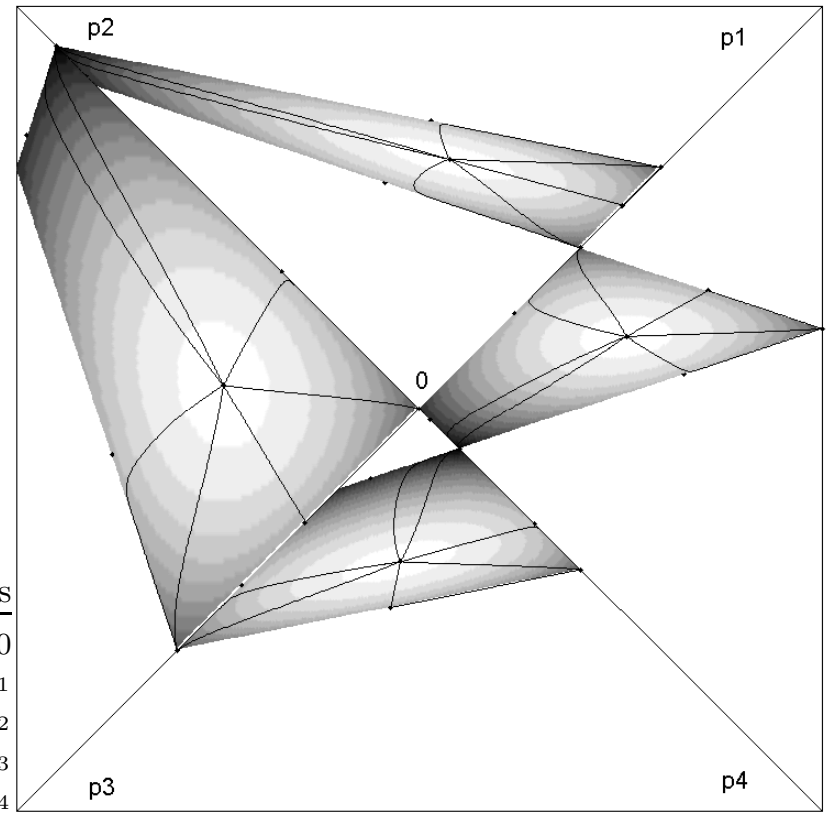

FIG. 7. Each rotated quadrant of the graph represents, for the corresponding pair of occupation probabilities, a plot of the seven trajectories shown in Figure 6 drawn over contour plots of the entropy surface.

\section{CONCLUSIONS}

The model we propose for the description of the time evolution of the occupation probabilities of a perturbed, isolated, physical system with single-particle eigenstates with energies $e_{i}$ for $i=1,2, \ldots, N$, is in good agreement with general thermodynamic requirements such as energy conservation, conservation of normalization and non-negativity of the probabilities, entropy nondecrease, E-conditional stability of the maximal-entropy canonical equilibrium states, $E$-conditional non-stability of each non-maximal-entropy partially-canonical equilibrium states, and existence and uniqueness of solutions for all initial perturbed distributions, both in forward and backward time. As in our previous work [17,19,20,23,26], the proposed rate equations implement the fundamental ansatz that nonequilibrium time dependence follows the path of steepest-entropy-ascent (or, using the terminology adopted in $[2,4]$, maximal entropy generation).

The model can be readily generalized to include additional constraints and therefore adapted to other physical and nonphysical (e.g., information theoretical, biological) problems that obey the same maximal entropy formalism and the maximal entropy generation rate ansatz. Using the formalism developed in Section III it can even be readily generalized to different entropy functionals or nonlinear objective functionals that may be relevant in many other contexts that share with the present the basic mathematical framework.
[1] M. Lemanska and Z. Jaeger, Physica D 170, 72 (2002).

[2] S. Gheorghiu-Svirschevski, Phys. Rev. A 63, 054102 (2001).

[3] R.F. Simmons and J. L. Park, Found. Phys. 11, 297 (1981).

[4] S. Gheorghiu-Svirschevski, Phys. Rev. A 63, 022105 (2001).

[5] G. Domokos and S. Kovesi-Domokos, J. Phys. A 32, 4105 (1999).

[6] M. Czachor, Phys. Rev. A 57, 4122 (1998); M. Czachor and M. Kuna, ibid. 58, 128 (1998); M. Czachor and J. Naudts, Phys. Rev. E 59, R2497 (1999).

[7] For an account of the vaste literature on these applications see the following papers and references therein: S. Weinberg, Phys. Rev. Lett. 62, 485 (1989); A. Stern, Y. Aharonov, and Y. Imry, Phys. Rev. A 41, 3436 (1990); A. K. Ekert, Phys. Rev. Lett. 67, 661 (1991); J. A. Holyst and L. A. Turski, Phys. Rev. A 45, 6180 (1992); G. Vidal and R.F. Werner, ibid. 65, 032314 (1993); W. G. Unruh and R. M. Wald, Phys. Rev. D 52, 2176 (1995); C. H. Bennett et al., Phys. Rev. Lett. 76, 722 (1996); M. Grigorescu, Physica A 256, 149 (1998); A. Miranowicz, H. Matsueda and M. R. B. Wahiddin, J. Phys. A 33, 5159 (2000); L. Hackermüller, K. Hornberger, B. Brezger, A. Zeilinger and M. Arndt, Nature, 427, 711 (2004).

[8] J. L. Park, Found. Phys. 21, 83 (1991).

[9] J. L. Park and W. Band, Found. Phys. 22, 657 (1992).

[10] G. N. Hatsopoulos and E. P. Gyftopoulos, Found. Phys. 6, 15, 127, 439, 561 (1976).

[11] K.F. Freed and D.F. Heller, J. Chem. Phys. 61, 3942 (1974); V.M. Kenkre and V. Seshadri, Phys. Rev. A 15, 197 (1977); V.M. Kenkre, ibid. 16, 766 (1977); V. Seshadri and V.M. Kenkre, ibid. 17, 223 (1978); and subsequent literature: e.g., S. Yang, J. Shao, and J. Cao, J. Chem. Phys. 121, 11250 (2004); H. Gassmann, F. Marquardt, and C. Bruder, Phys. Rev. E 66, 041111 (2002); U. Kleinekathöfer, et al., ibid. 66, 037701 (2002); S. Yan, F. Sakata, and Y. Zhuo, ibid. 65, 031111 (2002); Y.V. Palchikov, et al., ibid. 66, 016122 (2005); A. Shabani and D.A. Lidar, Phys. Rev. A 71, 020101(R) (2005).

[12] A. Royer, Phys. Rev. Lett. 77, 3272 (1996); W.T. Strunz, L. Diósi, and N. Gisin, ibid. 82, 1801 (1999); T. Yu, L. Diósi, and N. Gisin, and W.T. Strunz, Phys. Rev. A 60, 91 (1999); A. Royer, Phys. Lett. A 315, 335 (2003).

[13] H.P. Breuer, B. Kappler, and F. Petruccione, Phys. Rev. A 59, 1633 (1999).

[14] G.P. Beretta, Mod. Phys. Lett. A 20, 977 (2005).

[15] See, e.g., E. P. Gyftopoulos and G. P. Beretta, Thermodynamics: Foundations and Applications, Macmillan, New York, 1991 or Dover Publications, Mineola, NY, 2005.

[16] G. P. Beretta, J. Math. Phys. 27, 305 (1986). The technical conjecture proposed in this paper was later found proved in F. Hiai, M. Ohya, and M. Tsukada, Pacific J. Math. 96, 99 (1981).

[17] G. P. Beretta, E. P. Gyftopoulos, J. L. Park, and G. N. 
Hatsopoulos, Nuovo Cimento B 82, 169 (1984).

[18] G. P. Beretta, E. P. Gyftopoulos, and J. L. Park, Nuovo Cimento B 87, 77 (1985).

[19] G. P. Beretta, in Frontiers of Nonequilibrium Statistical Physics, proceedings of the NATO Advanced Study Institute, Santa Fe, June 1984, edited by G. T. Moore and M. O. Scully (NATO ASI Series B: Physics 135, Plenum Press, New York, 1986), p. 193 and p. 205; G. P. Beretta, "Steepest entropy ascent in quantum thermodynamics," in The Physics of Phase Space, edited by Y.S. Kim and W. W. Zachary (Lecture Notes in Physics 278, SpringerVerlag, New York, 1986), p. 441.

[20] G. P. Beretta, Int. J. Theor. Phys. 24, 119 (1985).

[21] G. P. Beretta, Int. J. Theor. Phys. 24, 1233 (1985).

[22] H. J. Korsch and H. Steffen, J. Phys. A 20, 3787 (1987); M. Hensel and H. J. Korsch, J. Phys. A 25, 2043 (1992).

[23] G.P. Beretta, "On the General Equation of Motion of Quantum Thermodynamics and the Distinction between Quantal and Nonquantal Uncertainties", Sc. D. thesis, M.I.T., 1981, available at arXiv:quant-ph/0509116.

[24] J. Maddox, "Uniting mechanics and statistics", Nature 316, 4 July 1985.

[25] T.S. Kuhn, The Structure of Scientific Revolutions, The University of Chicago Press, Chicago, 1970.

[26] G. P. Beretta, Found. Phys. 17, 365 (1987).

[27] That the relaxation time $\tau$ needs not be constant (as was initially assumed in $[17,18,20,21,23]$ as well as in [1]) but may be any positive functional of $\mathbf{p}$ was first realized in $[26]$.

[28] The $\rho=\gamma \gamma^{\dagger}$ formalism proposed by B. Reznik, Phys. Rev. Lett. 76, 1192 (1996) and adopted by GheorghiuSvirschevski [4], does extend the $\rho=(\sqrt{\rho})^{2}$ formalism first introduced in $[23,17,19]$ in an interesting and elegant way, however, it is not necessary to derive our nonlinear equation. Moreover, it may be conceptually troublesome because it leaves unspecified the choice of $\gamma$ for a given $\rho$, and thus risks to imply the existence of yet unidentified (hidden?) physical observables that would render the operator $\rho$ an insufficient state descriptor.

[29] As we discuss in a forthcoming paper to be published elsewhere, the important relation (28) holds also for the quantum equation proposed in $[23,4,17]$, i.e., also when the restriction $[H, \rho]=0$, which defines the class of distributions considered in the present paper, is removed. 